Clin Obstet Gynecol. 2018 September ; 61(3): 419-432. doi:10.1097/GRF.0000000000000389.

\title{
Management of the Perimenopause
}

\author{
Lara Delamater, MD and Nanette Santoro, MD* \\ University of Colorado School of Medicine Department of Obstetrics and Gynecology, $12631 \mathrm{E}$ \\ $17^{\text {th }}$ Avenue, Mail Stop B-198, Aurora, Colorado 80045
}

\begin{abstract}
Perimenopause, or the menopausal transition, represents a period of time during which newly arising symptoms can present complex management decisions for providers. Many women present to care with complaints of hot flashes, vaginal and sexual changes, altered mood and sleep, and changing bleeding patterns. The effect of these symptoms on quality of life, even before a woman enters menopause, can be significant. The appropriate evaluation and evidence-based management of women in this transition is reviewed in this article. Two case vignettes are used to highlight certain evaluation and treatment challenges.
\end{abstract}

\section{Keywords}

Perimenopause; menopause transition; hot flashes; abnormal uterine bleeding

\section{Introduction and Case Vignettes}

Perimenopause, or the menopausal transition, encompasses that period of time during which physiologic changes mark progression toward a woman's final menstrual period (FMP). This phase begins with the onset of menstrual irregularities and continues until a woman reaches menopause, or one year after amenorrhea has occurred ${ }^{1}$. Perimenopause, then, can last for a variable amount of time, the median of which is four years. Changing hormone levels manifest in varying symptoms that can present complex clinical management situations for providers. As many as $90 \%$ of women will present to health care providers for advice on how to cope with menopausal symptoms, demonstrating the importance of this issue worldwide ${ }^{2}$.

Understanding the physiology of the decline in ovarian function, which results in systemic hormonal changes and ultimately, symptoms, can help guide management. Traditionally, perimenopause includes an early and a late stage: the early stage is defined by occasional skipped cycles. The second stage is characterized by greater menstrual irregularity, with periods of amenorrhea lasting over 60 days and up to 12 months. Twelve or more months of amenorrhea defines the FMP, after which future menses are very unlikely ${ }^{3}$. The purpose of this review is to explore these stages of the menopausal transition and associated hormonal

*Corresponding author: Phone 303-724-2041, FAX: 303-724-2061, Nanette.Santoro@ ucdenver.edu. 
events, review common symptoms that characterize this period, and provide in depth evidence-based recommendations for evaluation and treatment.

\section{Case \#1}

Ms. M is a 45 year old woman who has been using oral contraceptives since the birth of her second child 10 years ago. She has noted progressively heavier menses during the pill free week over the past 9 months, with menstrual cramping that is only partially relieved by ibuprofen 600-800mg tid, and hot flashes during her menstrual periods. She also reports a feeling of pelvic heaviness. On bimanual examination, her uterus is about 8-10 weeks' size with an irregular contour. She requests an endometrial ablation, because her friend reported complete cessation of menses from this procedure. She does not believe she needs contraception any longer, because she is now 'close to menopause.'

Case \#2

Ms. Y is a 50 year old woman who had her last menstrual period 4 months ago, and reports having moderate- to-severe hot flashes that are particularly bothersome at night. She is the CEO of a regional pharmaceutical company, and has noted that her frequent travel related to her work has become 'exhausting' due to the chronic sleep disruption. She also reports experiencing anxiety when she speaks publicly for fear of having a hot flash mid-speech. She has a BMI of $22 \mathrm{~kg} / \mathrm{m}^{2}$ and reports taking $12.5 \mathrm{mg}$ of hydrochlorthiaziide daily for 'borderline' elevated blood pressure. In the office, her BP is 150/90, which she attributes to a stressful drive with 'too much traffic'.

\section{Questions}

- What additional information on history and physical exam would be helpful in assessing this patient?

- What evaluation is indicated at this time?

- $\quad$ How would you counsel this patient about the natural history of menopause and how it may impact her symptoms and treatment options?

- What treatment options would you recommend?

- How do you plan to follow her through her menopausal transition?

\section{Epidemiology}

Ongoing research has enhanced our understanding of the epidemiology of the menopausal transition ${ }^{4}$. With the goal of establishing nomenclature and standardized reporting for clinical and research purposes, the first Stages of Reproductive Aging Workshop (STRAW) in 2001 developed a map of both hormonal and clinical changes preceding and following the FMP to define perimenopausal stages. ${ }^{5}$ This paradigm was reevaluated by a multidisciplinary team in 2011 (ReSTAGE), with a follow up workshop, including support of further data from multiple cohorts, incorporating the interval information on hormonal changes ${ }^{1}$. Classifications from this workshop confirmed the division of the menopausal transition into an early and a late stage. Figure 1 details the criteria and important 
characteristics of these stages. The early menopausal transition is defined as either a persistent difference of 7 days or more in the length of consecutive cycles, or one more skipped cycles (persistence, in this case, is demonstrated by a recurrence in this cycle length difference that occurs within the subsequent 10 cycles). This irregularity progresses to longer periods of amenorrhea; when they last 60 days or longer, the patient has moved to the late menopausal transition, marked by increasing duration of amenorrhea, and more dramatic fluctuations in hormones. This late stage is more likely to be associated with vasomotor symptoms (VMS), although the highest VMS prevalence is found after the FMP. An individual patient's menopausal transition may not always follow this staging precisely. For example, some women, particularly those who transition at a later age, may skip the early menopause transition stage entirely; others, particularly those who transition at earlier ages, may spend many years in the early stage ${ }^{6}$. Traversal through the STRAW stages are also influenced by racial and ethnic differences, BMI, and lifestyle choices ${ }^{1}$. While many hormonal markers have been observed to change across the menopausal transition, none are presently useful for diagnostic purposes and staging remains best performed using menstrual history alone ${ }^{7}$. For women with irregular menses or amenorrhea (e.g., polycystic ovary syndrome), tracking the transition stages is not currently possible.

As women traverse menopause, symptoms and other health markers have been more closely evaluated through a number of studies. The Study of Women's Health Across the Nation (SWAN) represents an ethnically diverse cohort of North American Women, addressing a lack of diversity in previous studies. Through this cohort, the Melbourne Women's Health Study, and the Penn Ovarian Aging Study among others, we have come to better understand both the hormonal changes as well as the symptoms that constitute the transition. The menopausal transition encompasses a median of four or more years, with median onset at age 47, progression to late menopausal transition at a median age of 49 and arrival at the FMP at age $51-52^{8}$. This progression varies according to the age at onset of the menopausal transition, with a cohort of earliest onset symptoms translating to a longer median time of transition (8.6 years), as compared to a late onset symptoms cohort demonstrating a shorter median time of transition (4.3 years). SWAN has demonstrated that transit time to menopause can be impacted by other factors; for instance, African American women had a longer duration than white women, and greater body mass index was associated with a later onset menopausal transition.

\section{Hormonal changes}

The physiologic changes of the menopausal transition stem primarily from the decline in ovarian function. Various markers of ovarian aging have emerged to determine transitions within the perimenopause. Traditionally, FSH has been utilized as a measure of ovarian aging, but assessing this gonadotrophin has its limitations given its indirect correlation with ovarian functional decline. Although determining perimenopausal status relates to the patient's age, menstrual history and experience of VMS, further evaluation in the menopausal transition can also include other laboratory information such as estradiol, and Anti-mullerian hormone. Here we review briefly the timing of hormonal events throughout the menopausal transition, and the factors that influence them. 


\section{Early to Late Changes}

Hormone changes mark the progression through the menopausal transition, but can be highly variable and not always correlative with clinical symptoms. For instance, FSH is often, but not always, elevated even prior to onset of menstrual cycle irregularity, sometimes even 10 years before onset of the menopausal transition. A popular but incorrect belief is that the dwindling supply of ovarian follicles that leads to menopause manifests during the transition as a progressive decrease in estradiol. The earliest detectable event in the menopausal transition, as identified by Burger, et al, is a decrease in inhibin ${ }^{9}$. Inhibin is a peptide of the transforming growth factor-beta (TGF-B) family, produced by the granulosa/ luteal cells of the ovary. The physiologic role of inhibin (which exists in two forms, A and B) is to reduce pituitary FSH secretion via negative feedback inhibition ${ }^{10}$. In the early transition, when the follicle cohort decreases, inhibin B declines, leading to a loss of pituitary inhibition, and FSH secretion is increased, albeit intermittently. Thus, the rise in FSH in the early transition is not due to decreased estradiol ${ }^{11,12}$. Falls in estradiol are more characteristic of the late menopausal transition, as the number of anovulatory cycles increase. Increases in FSH and decreases in inhibin B generally represent early changes, and are followed by decreases in both estradiol and inhibin A in the late stage of menopausal transition; however, significant variability is emphasized in all such reviews ${ }^{13}$. Given this variability, although commonly performed, assessing FSH levels is not recommended as a test to determine if a patient is perimenopausal.

\section{Anti-mullerian Hormone}

Anti-mullerian hormone (AMH), like inhibin, is a TGF-B family peptide that is produced by the small, growing follicles of the ovary and is a biologically plausible marker of ovarian reserve ${ }^{14}$. AMH has been found to decrease over the reproductive life span and to directly reflect ovarian follicular reserve ${ }^{15}$. As women approach menopause, AMH decreases, and two AMH determinations, used to determine the rate of change, have been found to predict the age at menopause within about 4 years ${ }^{16}$, only slightly better than what can be determined using menstrual cyclicity alone. When more sensitive AMH assays have been used, the predictive value of AMH increases, but such measurements are not currently available for clinical practice ${ }^{17}$. Another recent analysis of a population based cohort from the US indicated that an AMH $200 \mathrm{pg} / \mathrm{ml}$ provided very strong predictive value that the FMP would NOT occur within the next 5 years ${ }^{18}$. AMH has been demonstrated to be more accurate in predicting time to the FMP than FSH, estradiol, or inhibin and is largely independent of the patient's BMI, race or ethnicity ${ }^{17}$. While it remains a promising marker, $\mathrm{AMH}$ is not currently recommended as a routine diagnostic test for perimenopause.

\section{Other Markers}

Longitudinal studies of menstrual cycle hormones using measurement of urinary metabolites reveals a progressive decline in urinary progesterone metabolites across the menopausal transition ${ }^{19,20}$. However, the degree of decline is relatively small and again, not helpful for diagnostic purposes. The proportion of cycles with low or anovulatory progesterone production appears to increase slightly as women traverse the menopause, but anovulatory cycles do not become markedly more prevalent until the 2 years before the final menses ${ }^{20}$. A 
common misconception about the menopausal transition is that androgens decline precipitously over this time period. Multiple longitudinal cohort studies do not confirm this notion. Although testosterone secretion drops with menopause, a concomitant decline in SHBG leads to either maintenance or even an increase in the free androgen index ${ }^{21}$. Declines of SHBG of $43 \%$ and increases in free androgen index of $80 \%$ have been reported $^{21}$. Adrenal androgens decline throughout adulthood, and continue this decline throughout the transition ${ }^{22,23}$. However, there is a transient rise in DHEAS in the latter transition in a majority of women who have been followed longitudinally ${ }^{24}$. Taken together, the available data do not favor the notion that androgen deficiency is a common feature of the menopause transition.

\section{Factors Influencing Hormone Levels}

BMI and race impact hormones. Obesity is associated with lower LH, FSH, estradiol and progesterone secretion, as well as marked decreases in urinary hormone metabolite excretion ${ }^{11}$. Women with obesity have a lower rise in FSH and a less steep decline in estradiol as they traverse the FMP ${ }^{25}$. African American and Hispanic women had higher FSH and lower testosterone compared to other ethnic groups based on an analysis of 2930 women from the SWAN Study ${ }^{26}$.

In addition to BMI and race, which account for variability in hormones over time, there are cycle to cycle changes that may impact the menopause and symptom experience. For example, women who experience the cycle irregularity of the early menopause transition at a younger age than the median of 47 are more likely to have a longer, and more symptomatic transition than those whose onset is later in life ${ }^{6}$. Women who have more variable hormone levels over time are also more likely to have a symptomatic menopause transition ${ }^{27}$.

\section{Symptoms}

\section{Vasomotor Symptoms}

Vasomotor symptoms (VMS), also known as hot flashes or hot flushes, are described as sudden onset, intense feelings of warmth often involving the face, neck or chest, sometimes followed by a chill. The longstanding theory exists that VMS are related to the withdrawal of estrogen, specifically estradiol. Interestingly, ambient serum estradiol does not correlate with presence or severity of symptoms; the one marker independently associated with increased risk of VMS was higher levels of $\mathrm{FSH}^{28}$. More clearly, VMS appear to be related to a complex combination of genetic makeup, physical changes and other outside influences, including culture and expectations.

It appears that up to $80 \%$ of women experience VMS during the perimenopause, increasing over the transition from early to late menopausal transition. The cohort of women in the SWAN study reported a prevalence of $39 \%$ in the early transition progressing to $67 \%$ cumulative prevalence ${ }^{29}$. Those women who were pre or perimenopausal at the onset of their reported VMS were also demonstrated to have the longest duration of symptoms and persistence after their FMP, with a median of 11.8 years, as compared to those who were postmenopausal at the time of onset (median duration of 3.4 years) ${ }^{30}$. Overall, while VMS 
previously were thought to last for under two years, these studies have more recently demonstrated median durations of 7 to 10 years, with African American women found to have the longest median duration of 10.1 years compared to 8.9 years for Hispanic women, 6.5 years for non-Hispanic white women, 5.4 years for Chinese women and 4.8 years for Japanese women ${ }^{30,31}$. Other factors that impact risk and severity include BMI, cigarette smoking, depressive symptoms, and alcohol intake ${ }^{29}$. In the SWAN study, lower rates of hot flash reporting were noted in Chinese and Japanese women. A protective effect in duration among women reporting 12 or more alcoholic drinks in the previous year has also been reported $^{32}$. Finally, women with a high body-mass index noted worse VMS during the menopausal transition, but milder symptoms after the FMP ${ }^{33}$.

Given that a significant cause of mortality in women is cardiovascular disease (CVD), investigators have sought to determine if VMS might represent a biomarker for its risk. One large study noted that women having frequent VMS had an increased risk of developing CVD over a 14 year period; this finding was demonstrated in women reporting later onset VMS as compared to those experiencing them in the early menopausal transition ${ }^{34,35}$ The crossover between these symptoms and cardiovascular risk highlights the importance of assessing women for CVD risk factors early in the clinical relationship, ideally even before onset of VMS.

\section{Vaginal and Sexual Symptoms}

Vaginal dryness, atrophy and associated symptoms such as dyspareunia and urinary symptoms, are common in the postmenopause, affecting between $1 / 3$ to $1 / 2$ of women or more $^{36}$. Symptoms may also arise in the perimenopause. These symptoms, collectively, are termed genitourinary syndrome of menopause and are associated with decreased vaginal collagen and elastin content, thinned epithelium, and increased vaginal $\mathrm{pH}$ related to decreasing estrogen ${ }^{37}$. Vaginal dryness and its effect on quality of life, particularly its impact on sexual function, is of significant import to patients. Cross-sectional studies have suggested that increased pain during sexual intercourse may occur earlier in the transition, while decreases in desire may occur later in a woman's transition. The SWAN cohort noted a decrease in reported sexual functioning starting at around 20 months prior to the FMP, with the greatest rate of decline in the late transition and a slower decline in postmenopause ${ }^{38}$. Of women reporting vulvovaginal symptoms, $40 \%$ also reported overall sexual dysfunction ${ }^{39}$. However, the most important factors influencing sexual function were not hormonal determinants, but rather prior, premenopause transition level of sexual function and relationship satisfaction ${ }^{40}$. While many perimenopausal symptoms such as VMS and mood tend to improve with time, ongoing targeted treatment is required for improvement in vaginal symptoms (please see chapter on Genitourinary Syndrome of Menopause).

\section{Sleep and Mood Changes}

Sleep is a very common concern among women in the menopause transition. Trouble falling asleep, early awakening, and interrupted sleep can occur. In the Study of Women Across the Nation, $37 \%$ of women between the ages of 40 and 55 reported difficulty sleeping, with highest rates noted in Caucasian and Hispanic women ${ }^{41}$. Sleep disturbances are strongly associated with vasomotor and psychological symptoms, in addition to stress- and health 
related lifestyle factors. With more severe VMS, a woman is more likely to report insomnia; however VMS alone do not explain all of the sleep changes during the transition. Hormone levels are not associated with sleep disturbances. Poor sleep prior to the transition is predictive of worsening sleep symptoms throughout menopause. ${ }^{41}$. Sleep symptoms tend to stabilize as a woman progresses to postmenopause ${ }^{42}$.

Sleep habits and presence of problems should be routinely assessed in clinic visits, given the significant impact on quality of life and health outcomes. A brief screen to determine the cause of the sleep problem is indicated. Women who have difficulty falling asleep (insomnia) can be screened with simple self-assessment tools ${ }^{43}$. Depression, which can cause early morning awakening, restless leg syndrome, and sleep apnea should also be ruled out prior to attributing sleep problems to estrogen deficiency.

The impact of the menopausal transition on mood is strongly related to quality of life during the period. Women should be routinely assessed for major depressive disorders and other psychiatric pathology as for any other cohort, noting that the risk of adverse mood symptoms (based on the Center for Epidemiologic Studies-Depression scale) increased from $20 \%$ to $62 \%$ by the early perimenopause as compared to premenopause. In the SWAN cohort, prevalence of psychological distress (CES-D score $\geq 16$ ) was $28.9 \%$ in the early menopausal transition, $25.6 \%$ in the late menopausal transition, and $22 \%$ in postmenopause $^{44}$. Three robust longitudinal studies-SWAN, the Penn Ovarian Aging Study, and the Harvard Study of Moods and cycles all demonstrated an increased risk for depressed mood during the menopausal transition, ${ }^{44-46}$ including increased risk of new-onset major depressive disorder and anxiety in women with no prior history of a psychologic diagnosis. Major depressive episodes are defined by DSM V as having 5 or more symptoms (weight changes or change in appetite, insomnia or hypersomnia, psychomotor agitation or retardation, fatigue or loss of energy or interest, feelings of worthlessness or guilt, decreased concentration, recurrent thoughts of death), one of which must include either depressed mood or loss of interest, causing significant distress or impairment in social, occupational, or other areas of functioning ${ }^{47}$. Using this definition, peri- and postmenopausal women were 2-4 times more likely to experience an episode, even when controlling for prior history of major depression, medication use, VMS, and very upsetting life events ${ }^{44}$.

\section{Bleeding}

Bleeding patterns among women in the perimenopause vary, thus distinguishing between "normal" and "abnormal" can be difficult. In the early menopause transition, short cycle intervals (fewer than 21 days) are more common, while long cycle intervals (more than 36 days) occur later in the transition ${ }^{48}$. During the menopausal transition, women were more likely to experience an increased number of days of bleeding, with 77\% reporting at least 3 episodes of $10+$ days of bleeding 49 .

Abnormal uterine bleeding presents a unique challenge to the provider in the perimenopause, as physiologic hormonal changes may mimic, or mask pathologic diagnoses. These presentations often warrant further evaluation to rule out serious conditions such as hyperplasia or cancer, and to guide treatment options. For perimenopausal women, 
the PALM-COEIN classification of abnormal uterine bleeding applies and should be utilized to establish a differential diagnosis of bleeding and to guide evaluation and management ${ }^{50}$.

Evaluation should include a thorough menstrual, medication, and family history, and assessment for endocrine dysfunction or bleeding disorders (when indicated). Examination should evaluate BMI, thyroid, uterus and cervix via both a speculum and bimanual assessment. This information will help guide laboratory testing, which may include complete blood count, iron studies, thyroid assessment, or evaluation for coagulation disorders. Transvaginal ultrasonography is the imaging modality of choice to identify uterine and endometrial structural abnormalities, although further assessment with saline infusion sonohysterography (SIS) or office hysteroscopy may be warranted for evaluation of intracavitary lesions ${ }^{51}$. Endometrial biopsy is indicated for persistent abnormal uterine bleeding, or in the perimenopausal setting for those with risk factors, including chronic anovulation and obesity. While endometrial biopsy is sensitive in detecting global endometrial pathology, it is important to keep in mind that its sensitivity decreases for focal pathology 52 . Once evaluation is complete, tailored management options can be discussed.

\section{Evidence-Based Management}

Management should take into account the bothersomeness of each symptom, and a holistic, yet economical approach is recommended. For example, rather than treating multiple symptoms with multiple medications, a single treatment is preferred. Sometimes concurrent medical problems can be addressed, and the treatment to be deployed will allow a patient to discontinue one of her current medications. This is another desirable approach. For example, a patient who is taking a hypnotic for sleep but complains of night sweats that are disrupting her sleep may benefit from a trial of gabapentin, which may simultaneously reduce her night sweats and promote uninterrupted sleep. This approach can allow her to discontinue the hypnotic. A simplified approach to management of common menopausal symptoms is provided in Figures 2 and 3.

Women who are not yet menopausal, but who have menopausal symptoms, add the additional challenge to the clinician of assuring that treatment does not cause irregular menstrual bleeding and that contraceptive needs are addressed. For these reasons, hormonal management with oral contraceptives or hormonal contraceptives in patch or ring forms are useful and FDA approved for use up to the age at menopause. There is evidence that lower dose estrogen preparations are $\operatorname{safer}^{54}$, and for this reason, the lower dose formulations containing 20 to $30 \mathrm{ug}$ of ethinyl estradiol are recommended as a starting point. Women at high risk for cardiovascular disease should be directed to alternative treatments, as the risk of myocardial infarction and stroke rises with age, and exposure to hormonal contraception increases the relative risk of an adverse cardiovascular event to 1.6 (95\% CI 1.2, 2.1 $)^{54}$. For these women, and for those who do not tolerate and do not wish to use systemically administered hormonal contraception, an intrauterine device, with or without progestin, is an effective alternative. Progestin-containing intrauterine devices (IUDs), although not approved for use in menopausal women, appear to provide ample, local progestin, and can allow the clinician to provide exogenous estrogen as needed to control VMS and other symptoms ${ }^{55,56}$. The progestin-containing IUD has the added advantage of reducing or 
eliminating menstrual bleeding entirely, and has therapeutic value for common gynecological problems of women in their 40's, such as adenomyosis. Additionally, tranexamic acid offers a non-hormonal mechanism for decreasing objective quantity of bleeding 57 .

\section{Conclusions}

Patients who are treated with HT with satisfactory control of symptoms often wonder when birth control can be safely discontinued. Unfortunately for the clinician, there is at present no foolproof way to assure a patient that she is rendered permanently infertile and is truly menopausal other than 12 months of observed amenorrhea off of all hormones. Many symptomatic women will not tolerate such a long period of time off HT. Others can be switched to a non-hormonal alternative and may choose to use barrier methods of contraception while they await the traditional 12 month definition of menopause. The median age at menopause in the United States is 51 years ${ }^{58}$. Therefore, half of all US women will be menopausal by this age and this is a reasonable point of departure for shared decision making about when to discontinue contraception. Hormonal testing to determine definitively when a woman is truly menopausal can be misleading and is not recommended, as current methods are not sufficiently sensitive or specific to provide confidence that they are correct. AMH may finally provide a test that is clinically useful, but it is still in the experimental phases ${ }^{17}$. Although the risk of conception is very low $(<0.1 \%)$ for women aged 44 and older ${ }^{59}$, hormonally normal-appearing menstrual cycles have been reported up to the end of reproductive life. ${ }^{20}$ The possibility of an unwanted conception in the late perimenopause remains a potential, but unlikely issue.

Patients who are satisfied with their HT can be reassured that, for the majority of women, VMS will eventually subside and will no longer require treatment. However, since the median duration of VMS can exceed 10 years, women may choose to wean off hormones sooner, or intervening health conditions may emerge that limit continued use of hormones. In these circumstances, non-hormonal alternatives can be tried if symptoms remain severe. For many women, it may be simple to wean off of HT. Hormones can be discontinued in a number of different ways. Pills (and patches) can be cut in half to slowly reduce the dose. It is important to remember that most oral HT preparations will be essentially eliminated over 24 hours; for this reason, alternate day dosing as a method of weaning is not recommended. Similarly, extending the time interval of a weekly estradiol patch beyond 7 days or a twiceweekly patch beyond 3.5 days in order to wean will result in an abrupt withdrawal from estradiol, as estradiol levels will plummet beyond the recommended dosing interval. Women should be warned that VMS are likely to return when HT is withdrawn, but they may be milder and will eventually subside. For the minority of women who continue to experience severe VMS well into the postmenopause, extended duration of HT may be considered after careful discussion of risks versus benefits ${ }^{60}$. Women who develop adverse effects from HT or who wish to discontinue may try non-hormonal alternative medications. 


\section{Management Considerations}

\section{Case 1}

Heavier menses are not expected to occur during a normal menopausal transition; rather, menses tend to get lighter over time ${ }^{48}$. The report of heavier bleeding in association with the patient's complaint of a mass effect should prompt a search for an anatomical lesion. Fibroids are suspected on the basis of her physical examination. Given the likelihood of fibroids, and the fact that the patient has experienced a change in pelvic pressure and increased bleeding, the clinician should consider the possibility that the fibroids are growing, and that a submucosal component is present. Accordingly, assessment of the uterus with vaginal ultrasound, saline infusion sonohystogram (SIS), or office hysteroscopy is appropriate. Complete menstrual suppression with continuous oral contraceptive pills (without a pill-free week) may successfully suppress menses ${ }^{61}$. If bleeding stops, this strategy may be a successful one over the long term. However, if breakthrough bleeding occurs and/or becomes heavy, a fibroid embolization may allow the clinician to temporize the bleeding and follow the patient through menopause without surgical intervention. If submucosal myomas are present, a hysteroscopic resection may take care of the bleeding without need for hysterectomy. If intramural/subserosal fibroids are present, endometrial ablation is unlikely to address the underlying problem causing the bleeding, e.g., fibroids. Should the above strategies not prove desirable or effective for this women, one final option for her to consider, in view of the increased pressure (bulky symptoms) as well as her abnormal bleeding, would be hysterectomy with ovarian conservation.

\section{Case 2}

This patient would likely benefit from low dose HT. Her elevated blood pressure is a caution, however, and may ultimately limit its use. Her blood pressure should be normalized, either with a second agent or by an increase in dose of her thiziade diuretic. Once her blood pressure is within normal range, weekly or bi-weekly transdermal estradiol $(25-50 \mathrm{mcg} / \mathrm{day})$ with nightly oral micronized progesterone $(100 \mathrm{mg})$ is likely to alleviate or eliminate her symptoms and restore more restful sleep. Blood pressure should be re-checked after hormones are initiated because estrogen can activate the renin-angiotensn-aldosterone system (RAAS) and thereby increase blood pressure further, although transdermal estradiol in the doses currently used for HT does not appear to activate the RAAS and may in fact lower $\mathrm{BP}^{62}$. Alternative hormonal regimens that can be considered would be conjugated equine estrogen or other oral estradiol or estrogen preparations, with nightly progesterone. Conjugated equine estrogen can also be given in combination with bazedoxifene to avoid use of progestin. This patient may also be treated with contraceptive doses of hormones, either oral, patch or vaginal ring, if contraception is needed. Contraceptive doses of hormones are substantially higher than those found in typical HT regimens-even the lowest dose options. Although they carry greater relative risk of cardiovascular complications with age, for most low-risk women the possibility of a major complication such as a heart attack remains small on an individual basis. For this patient under consideration, oral contraceptives or contraceptive doses may be less desirable. Use of a progestin IUD to provide contraception and deliver progestin to the uterus in combination with low dose 
estrogen, as described previously, if needed for treatment of hot flashes, may be a more desirable option. (Table)

\section{Acknowledgments}

Source of Funding: Dr. Santoro has is on the Scientific Advisory Board for Ogeda Pharmaceuticals and has stock options for Menogenix, Inc. For the remaining authors none were declared.

\section{References}

1. Harlow SD, Gass M, Hall JE, et al. Executive summary of the Stages of Reproductive Aging Workshop + 10: addressing the unfinished agenda of staging reproductive aging. J Clin Endocrinol Metab. 2012; 97:1159-68. [PubMed: 22344196]

2. Guthrie JR, Dennerstein L, Taffe JR, Donnelly V. Health care-seeking for menopausal problems. Climacteric. 2003; 6:112-7. [PubMed: 12841881]

3. Wallace RB, Sherman BM, Bean JA, Treloar AE, Schlabaugh L. Probability of menopause with increasing duration of amenorrhea in middle-aged women. Am J Obstet Gynecol. 1979; 135:10214. [PubMed: 517585]

4. Grady D. Clinical practice. Management of menopausal symptoms. N Engl J Med. 2006; 355:233847. [PubMed: 17135587]

5. Soules MR, Sherman S, Parrott E, et al. Stages of Reproductive Aging Workshop (STRAW). J Womens Health Gend Based Med. 2001; 10:843-8. [PubMed: 11747678]

6. Paramsothy P, Harlow SD, Nan B, et al. Duration of the menopausal transition is longer in women with young age at onset: the multiethnic Study of Women's Health Across the Nation. Menopause. 2017; 24:142-9. [PubMed: 27676632]

7. Burger HG. Unpredictable endocrinology of the menopause transition: clinical, diagnostic and management implications. Menopause Int. 2011; 17:153-4. [PubMed: 22120939]

8. Gold EB. The timing of the age at which natural menopause occurs. Obstet Gynecol Clin North Am. 2011; 38:425-40. [PubMed: 21961711]

9. Burger HG, Dudley EC, Robertson DM, Dennerstein L. Hormonal changes in the menopause transition. Recent Prog Horm Res. 2002; 57:257-75. [PubMed: 12017547]

10. Santoro N, Adel T, Skurnick JH. Decreased inhibin tone and increased activin A secretion characterize reproductive aging in women. Fertil Steril. 1999; 71:658-62. [PubMed: 10202875]

11. Santoro N, Lasley B, McConnell D, et al. Body size and ethnicity are associated with menstrual cycle alterations in women in the early menopausal transition: The Study of Women's Health across the Nation (SWAN) Daily Hormone Study. J Clin Endocrinol Metab. 2004; 89:2622-31. [PubMed: 15181033]

12. Santoro N, Brown JR, Adel T, Skurnick JH. Characterization of reproductive hormonal dynamics in the perimenopause. J Clin Endocrinol Metab. 1996; 81:1495-501. [PubMed: 8636357]

13. Hale GE, Zhao X, Hughes CL, Burger HG, Robertson DM, Fraser IS. Endocrine features of menstrual cycles in middle and late reproductive age and the menopausal transition classified according to the Staging of Reproductive Aging Workshop (STRAW) staging system. J Clin Endocrinol Metab. 2007; 92:3060-7. [PubMed: 17550960]

14. Kelsey TW, Wright P, Nelson SM, Anderson RA, Wallace WH. A validated model of serum antimullerian hormone from conception to menopause. PLoS One. 2011; 6:e22024. [PubMed: 21789206]

15. Santoro N. Using Antimullerian Hormone to Predict Fertility. JAMA. 2017; 318:1333-4. [PubMed: 29049571]

16. Freeman EW, Sammel MD, Lin H, Boorman DW, Gracia CR. Contribution of the rate of change of antimullerian hormone in estimating time to menopause for late reproductive-age women. Fertil Steril. 2012; 98:1254-9. e1-2. [PubMed: 22921911]

17. Finkelstein JS, Lee H, Burnett-Bowie S-A. , et al. Utility of Anti-Mullerian Hormone (AMH) for predicting the time to the final menstrual period: the Study of Women's Health Across the Nation 
(SWAN); Endocrine Society's 98th Annual Meeting; April 1-4, 2016; Boston, MA. 2016. OR216. (abstract)

18. Kim C, Slaughter JC, Wang ET, et al. Anti-Mullerian hormone, follicle stimulating hormone, antral follicle count, and risk of menopause within 5 years. Maturitas. 2017; 102:18-25. [PubMed: 28610678]

19. Santoro N, Crawford SL, Lasley WL, et al. Factors related to declining luteal function in women during the menopausal transition. J Clin Endocrinol Metab. 2008; 93:1711-21. [PubMed: 18285413]

20. Santoro N, Crawford SL, El Khoudary SR, et al. Menstrual Cycle Hormone Changes in Women Traversing the Menopause: Study Of Women's Health Across the Nation. J Clin Endocrinol Metab. 2017

21. Burger HG, Dudley EC, Cui J, Dennerstein L, Hopper JL. A prospective longitudinal study of serum testosterone, dehydroepiandrosterone sulfate, and sex hormone-binding globulin levels through the menopause transition. J Clin Endocrinol Metab. 2000; 85:2832-8. [PubMed: 10946891]

22. Rannevik G, Jeppsson S, Johnell O, Bjerre B, Laurell-Borulf Y, Svanberg L. A longitudinal study of the perimenopausal transition: altered profiles of steroid and pituitary hormones, SHBG and bone mineral density. Maturitas. 2008; 61:67-77. [PubMed: 19434880]

23. Lasley BL, Crawford SL, Laughlin GA, et al. Circulating dehydroepiandrosterone sulfate levels in women who underwent bilateral salpingo-oophorectomy during the menopausal transition. Menopause. 2011; 18:494-8. [PubMed: 21178790]

24. Crawford S, Santoro N, Laughlin GA, et al. Circulating dehydroepiandrosterone sulfate concentrations during the menopausal transition. J Clin Endocrinol Metab. 2009; 94:2945-51. [PubMed: 19470626]

25. Tepper PG, Randolph JF Jr, McConnell DS, et al. Trajectory clustering of estradiol and folliclestimulating hormone during the menopausal transition among women in the Study of Women's Health across the Nation (SWAN). J Clin Endocrinol Metab. 2012; 97:2872-80. [PubMed: 22659249]

26. Randolph JF Jr, Sowers M, Gold EB, et al. Reproductive hormones in the early menopausal transition: relationship to ethnicity, body size, and menopausal status. J Clin Endocrinol Metab. 2003; 88:1516-22. [PubMed: 12679432]

27. Freeman EW, Sammel MD, Lin H, et al. Symptoms associated with menopausal transition and reproductive hormones in midlife women. Obstet Gynecol. 2007; 110:230-40. [PubMed: 17666595]

28. Randolph JF Jr, Sowers M, Bondarenko IV, Harlow SD, Luborsky JL, Little RJ. Change in estradiol and follicle-stimulating hormone across the early menopausal transition: effects of ethnicity and age. J Clin Endocrinol Metab. 2004; 89:1555-61. [PubMed: 15070912]

29. Gallicchio L, Miller SR, Kiefer J, Greene T, Zacur HA, Flaws JA. Risk factors for hot flashes among women undergoing the menopausal transition: baseline results from the Midlife Women's Health Study. Menopause. 2015; 22:1098-107. [PubMed: 25783472]

30. Avis NE, Crawford SL, Greendale G, et al. Duration of menopausal vasomotor symptoms over the menopause transition. JAMA internal medicine. 2015; 175:531-9. [PubMed: 25686030]

31. Freeman EW, Sammel MD, Lin H, Liu Z, Gracia CR. Duration of menopausal hot flushes and associated risk factors. Obstet Gynecol. 2011; 117:1095-104. [PubMed: 21508748]

32. Smith RL, Gallicchio L, Miller SR, Zacur HA, Flaws JA. Risk Factors for Extended Duration and Timing of Peak Severity of Hot Flashes. PloS one. 2016; 11:e0155079. [PubMed: 27149066]

33. Thurston RC, Sowers MR, Sternfeld B, et al. Gains in body fat and vasomotor symptom reporting over the menopausal transition: the study of women's health across the nation. Am J Epidemiol. 2009; 170:766-74. [PubMed: 19675142]

34. van den Berg MJ, Herber-Gast GC, van der Schouw YT. Is an unfavourable cardiovascular risk profile a risk factor for vasomotor menopausal symptoms? Results of a population-based cohort study. BJOG. 2015; 122:1252-8. [PubMed: 25515605]

35. Szmuilowicz ED, Manson JE. Menopausal vasomotor symptoms and cardiovascular disease. Menopause. 2011; 18:345-7. [PubMed: 21266936] 
36. Santoro N, Komi J. Prevalence and impact of vaginal symptoms among postmenopausal women. J Sex Med. 2009; 6:2133-42. [PubMed: 19493278]

37. Kim HK, Kang SY, Chung YJ, Kim JH, Kim MR. The Recent Review of the Genitourinary Syndrome of Menopause. Journal of menopausal medicine. 2015; 21:65-71. [PubMed: 26357643]

38. Avis NE, Colvin A, Karlamangla AS, et al. Change in sexual functioning over the menopausal transition: results from the Study of Women's Health Across the Nation. Menopause. 2017; 24:379-90. [PubMed: 27801705]

39. Avis NE, Brockwell S, Randolph JF Jr, et al. Longitudinal changes in sexual functioning as women transition through menopause: results from the Study of Women's Health Across the Nation. Menopause. 2009; 16:442-52. [PubMed: 19212271]

40. Avis NE, Green R. The perimenopause and sexual functioning. Obstet Gynecol Clin North Am. 2011; 38:587-94. [PubMed: 21961721]

41. Kravitz HM, Joffe H. Sleep during the perimenopause: a SWAN story. Obstet Gynecol Clin North Am. 2011; 38:567-86. [PubMed: 21961720]

42. Kravitz HM, Janssen I, Bromberger JT, et al. Sleep Trajectories Before and After the Final Menstrual Period in The Study of Women's Health Across the Nation (SWAN). Curr Sleep Med Rep. 2017; 3:235-50. [PubMed: 28944165]

43. Buysse DJ, Rush AJ, Reynolds CF 3rd. Clinical Management of Insomnia Disorder. JAMA. 2017; 318:1973-4. [PubMed: 29059360]

44. Bromberger JT, Kravitz HM. Mood and menopause: findings from the Study of Women's Health Across the Nation (SWAN) over 10 years. Obstet Gynecol Clin North Am. 2011; 38:609-25. [PubMed: 21961723]

45. Cohen LS, Soares CN, Vitonis AF, Otto MW, Harlow BL. Risk for new onset of depression during the menopausal transition: the Harvard study of moods and cycles. Arch Gen Psychiatry. 2006; 63:385-90. [PubMed: 16585467]

46. Freeman EW, Sammel MD, Lin H, Nelson DB. Associations of hormones and menopausal status with depressed mood in women with no history of depression. Arch Gen Psychiatry. 2006; 63:375-82. [PubMed: 16585466]

47. First MB. Diagnostic and statistical manual of mental disorders, 5th edition, and clinical utility. J Nerv Ment Dis. 2013; 201:727-9. [PubMed: 23995026]

48. Van Voorhis BJ, Santoro N, Harlow S, Crawford SL, Randolph J. The relationship of bleeding patterns to daily reproductive hormones in women approaching menopause. Obstet Gynecol. 2008; 112:101-8. [PubMed: 18591314]

49. Paramsothy P, Harlow SD, Greendale GA, et al. Bleeding patterns during the menopausal transition in the multi-ethnic Study of Women's Health Across the Nation (SWAN): a prospective cohort study. BJOG. 2014; 121:1564-73. [PubMed: 24735184]

50. Munro MG, Critchley HO, Broder MS, Fraser IS, Disorders FWGoM. FIGO classification system (PALM-COEIN) for causes of abnormal uterine bleeding in nongravid women of reproductive age. Int J Gynaecol Obstet. 2011; 113:3-13. [PubMed: 21345435]

51. Goldstein SR, Lumsden MA. Abnormal uterine bleeding in perimenopause. Climacteric. 2017; 20:414-20. [PubMed: 28780893]

52. Committee on Practice B-G. Practice bulletin no. 128: diagnosis of abnormal uterine bleeding in reproductive-aged women. Obstet Gynecol. 2012; 120:197-206. [PubMed: 22914421]

53. Sherman S. Defining the menopausal transition. Am J Med. 2005; 118(Suppl 12B):3-7.

54. Roach RE, Helmerhorst FM, Lijfering WM, Stijnen T, Algra A, Dekkers OM. Combined oral contraceptives: the risk of myocardial infarction and ischemic stroke. Cochrane Database Syst Rev. 2015:CD011054. [PubMed: 26310586]

55. Somboonporn W, Panna S, Temtanakitpaisan T, Kaewrudee S, Soontrapa S. Effects of the levonorgestrel-releasing intrauterine system plus estrogen therapy in perimenopausal and postmenopausal women: systematic review and meta-analysis. Menopause. 2011; 18:1060-6. [PubMed: 21720280]

56. Santoro N, Teal S, Gavito C, Cano S, Chosich J, Sheeder J. Use of a levonorgestrel-containing intrauterine system with supplemental estrogen improves symptoms in perimenopausal women: a pilot study. Menopause. 2015; 22:1301-7. [PubMed: 26575111] 
57. Lethaby A, Farquhar C, Cooke I. Antifibrinolytics for heavy menstrual bleeding. Cochrane Database Syst Rev. 2000:CD000249.

58. Gold EB, Crawford SL, Avis NE, et al. Factors related to age at natural menopause: longitudinal analyses from SWAN. Am J Epidemiol. 2013; 178:70-83. [PubMed: 23788671]

59. Tietze C. Reproductive span and rate of reproduction among Hutterite women. Fertil Steril. 1957; 8:89-97. [PubMed: 13405050]

60. The NHTPSAP. The 2017 hormone therapy position statement of The North American Menopause Society. Menopause. 2017; 24:728-53. [PubMed: 28650869]

61. Maguire K, Westhoff $\mathrm{C}$. The state of hormonal contraception today: established and emerging noncontraceptive health benefits. Am J Obstet Gynecol. 2011; 205:S4-8. [PubMed: 21961824]

62. Seely EW, Walsh BW, Gerhard MD, Williams GH. Estradiol with or without progesterone and ambulatory blood pressure in postmenopausal women. Hypertension. 1999; 33:1190-4. [PubMed: 10334810] 


\section{Key Points}

- $\quad$ Perimenopause is defined as a period encompassing physiologic changes that result in the onset of menstrual irregularities and other symptoms, until a woman reaches menopause

- $\quad$ Commonly encountered symptoms in the perimenopausal period include hot flashes, vaginal or sexual symptoms, sleep and mood changes, and bleeding

- $\quad$ Menopausal symptoms may long precede the final menstrual period, and earlier onset may predict a longer duration of symptoms

- $\quad$ Evaluation and treatment of symptoms should be personalized based on type and severity of symptoms and contraceptive need, using a shared-decision making model 


\section{Early Menopausal Transition}

Persistent 7 day or more difference in length of consecutive cycles

\section{Late Menopausal Transition}

Interval of amenorrhea of 60 days or more, typically lasting $1-3$ years

\section{Postmenopause}

One year of amenorrhea

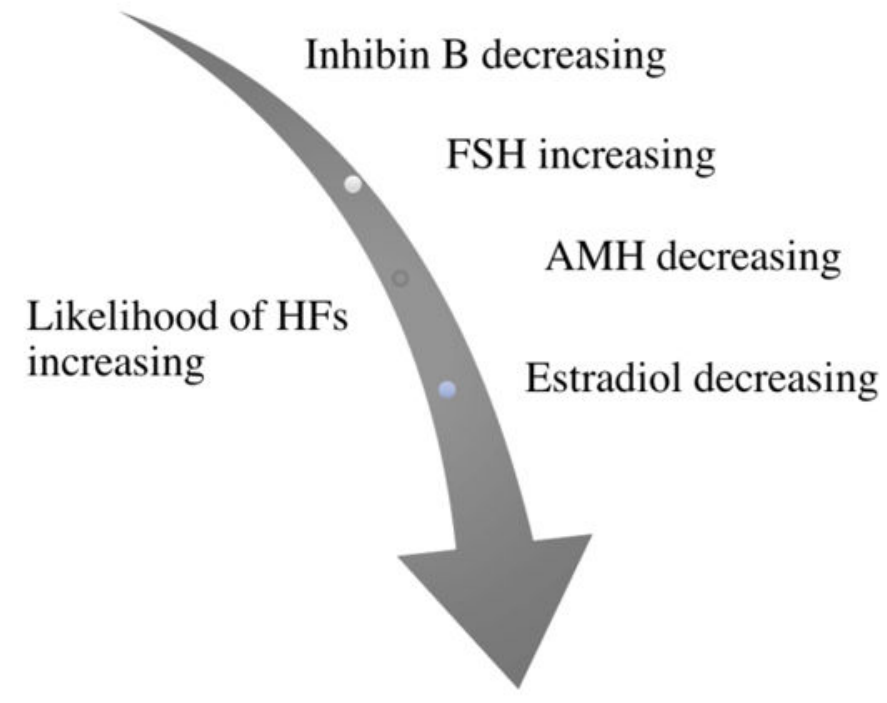

Figure 1.

Definitions and characteristics of the Stages of Reproductive Aging, simplified from the STRAW definitions. Progression through the perimenopause is marked by increasing menstrual cycle variability that serves to define these stages. As will be discussed, this correlates with trends in hormonal levels. In addition to bleeding pattern changes, symptoms are also significant for increasingly prevalent VMS. These typically begin either during the menopausal transition or early in the postmenopausal stage ${ }^{1}$. 

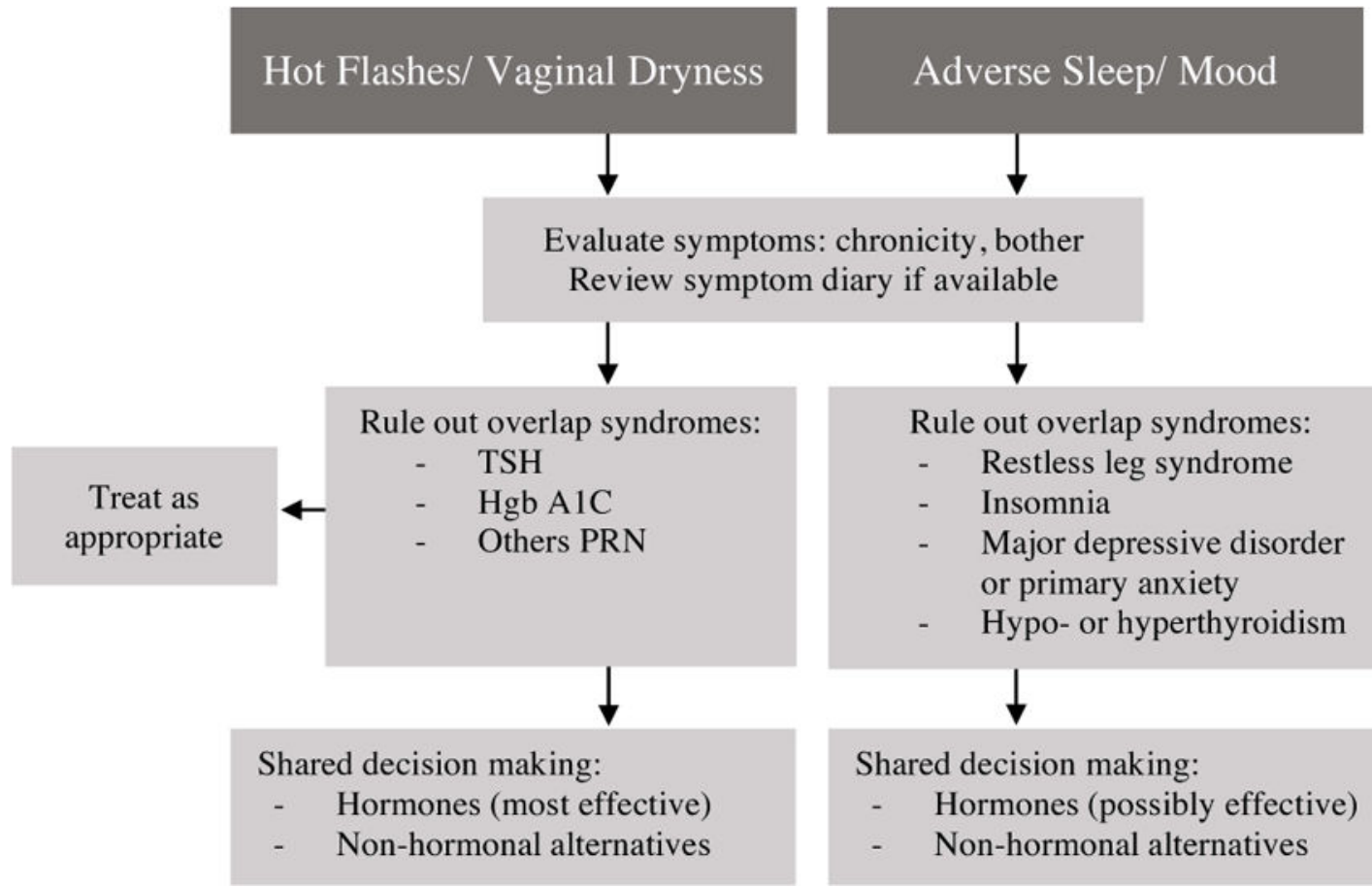

Evaluate symptoms: chronicity, bother Review symptom diary if available

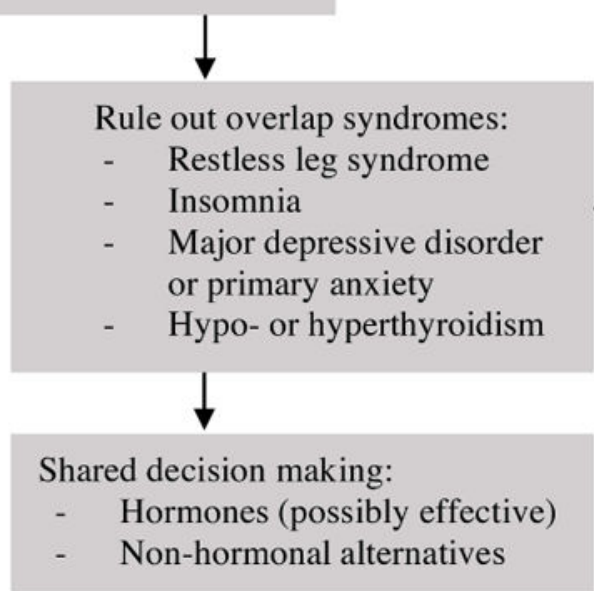

Figure 2.

Approach to evaluation and management of menopausal symptoms. Symptoms are grouped into two main categories, from among the 4 symptom sets that are most likely related to the menopausal transition ${ }^{53}$. Few symptoms mimic VMS, but because of their prevalence, ruling out thyroid disease and type 2 diabetes is prudent. Vaginal dryness can sometimes be due to conditions other than menopause (e.g. lichen sclerosus et atrophicus), and a physical examination is recommended. Restless leg syndrome and chronic insomnia often overlap with menopause-related sleep concerns, and treatment will be most effective if both problems are addressed. Primary mood disorders that are long-standing and/or severe are less likely to respond to hormone therapy (HT) alone; a thorough history will help the clinician determine whether single-agent therapy with hormones is indicated, or if comanagement with a mental health professional may be more effective. Hormones are likely the most efficient path to symptom control for women with VMS or vaginal dryness; in the latter case, topical hormones may be sufficient. Women with mood and sleep issues may benefit from a trial of hormones; if not sufficiently improved, additional, non-hormonal options or co-management should be considered. 


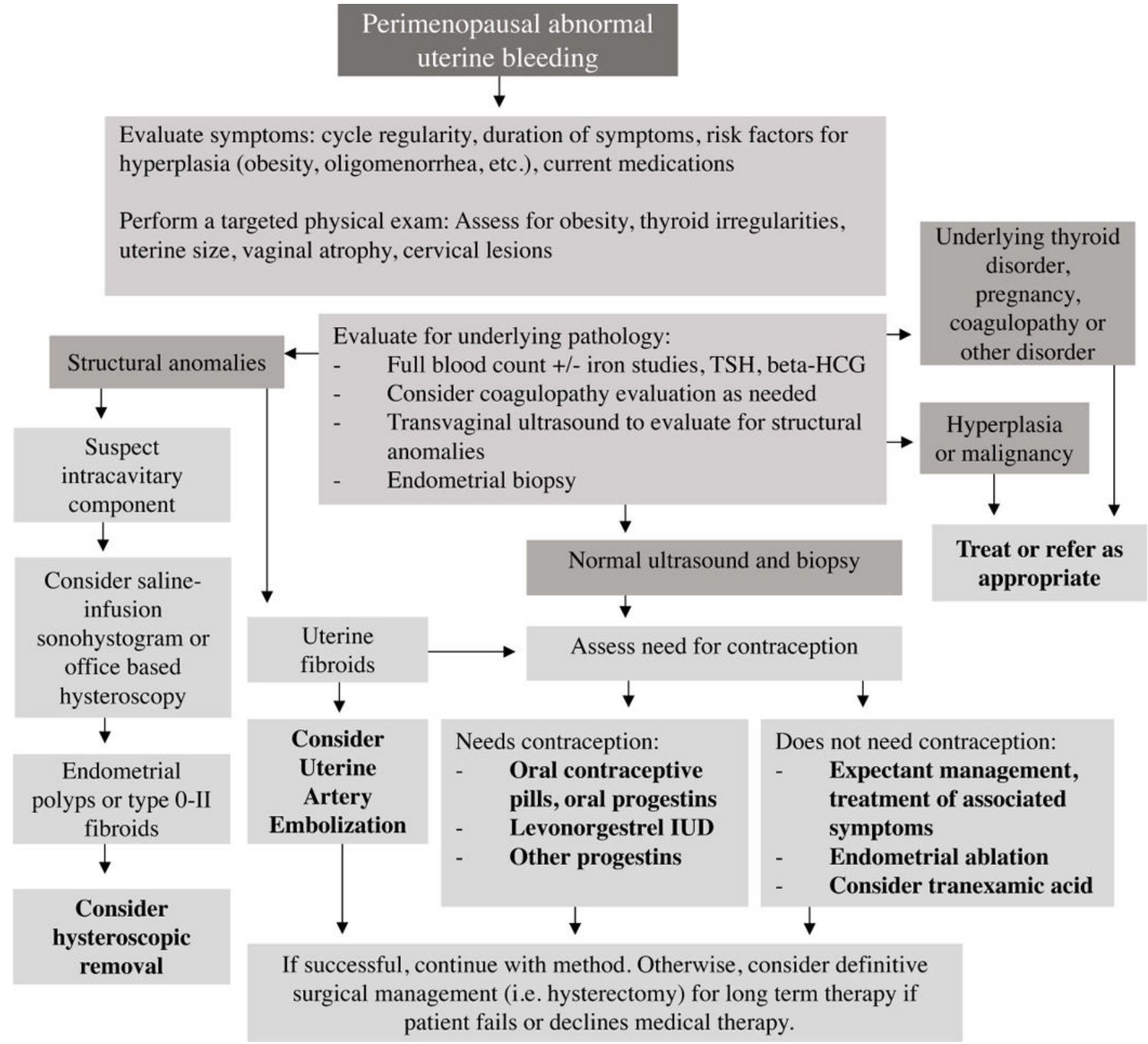

Figure 3.

Approach to management of abnormal uterine bleeding in perimenopausal patients, taking into account appropriate evaluation, need for contraception, and management options available. As shown, management should be targeted toward underlying pathology when encountered. Otherwise, decisions should incorporate a patient-centered approach after discussion of the patient's goals. It is important to note that, when workup has ruled out pathology necessitating medical intervention, reassurance can be provided to the patient and it is reasonable to pursue expectant management. Additionally, bleeding abnormalities may be multifactorial; anovulatory bleeding concurrent with structural abnormalities is less likely to resolve after addressing the structural component alone ${ }^{51}$. When symptoms are bothersome to the patient, treatment can range from medical to surgical management, and 
definitive surgical management (i.e. a hysterectomy) can be considered for patients who have failed or decline other methods. 
Table

Quality Of Evidence in Treatment of Symptoms in the Menopausal Transition

\begin{tabular}{|l|l|}
\hline \multirow{2}{*}{ Level III } & Grady D. Clinical practice. Management of menopausal symptoms. N Engl J Med 2006;355:2338-47. \\
\cline { 2 - 3 } & $\begin{array}{l}\text { Somboonporn W, Panna S, Temtanakitpaisan T, Kaewrudee S, Soontrapa S. Effects of the levonorgestrel-releasing intrauterine } \\
\text { system plus estrogen therapy in perimenopausal and postmenopausal women: systematic review and meta-analysis. Menopause } \\
2011 ; 18: 1060-6 .\end{array}$ \\
\cline { 2 - 3 } & $\begin{array}{l}\text { Committee on Practice Bulletins-Gynecology. Practice bulletin no. 128: diagnosis of abnormal uterine bleeding in reproductive- } \\
\text { aged women. Obstet Gynecol 2012;120:197-206. }\end{array}$ \\
\cline { 2 - 3 } & The 2017 hormone therapy position statement of The North American Menopause Society. Menopause 2017;24:728-53. \\
\hline
\end{tabular}

